\title{
Optical investigation of the electronic structure of alloys $\mathrm{Cu}-\mathrm{Fe}$
}

\author{
V.V. Vovchenko, V.S. Staschuk, L.V. Poperenko, V.O. Lysiuk \\ Department of Physics, Taras Shevchenko Kyiv National University \\ 2, Academician Glushkov prospect, 03022 Kyiv, Ukraine \\ Phone: +38 (044)5262296; fax:+38 (044)5264507; e-mail:plv@univ.kiev.ua
}

\begin{abstract}
We investigate the optical properties of $\mathrm{Cu}-\mathrm{Fe}$ alloys in a wide interval of concentrations (7.5-30 at. \%) and in a wide spectral interval $(0.25-7 \mu \mathrm{m})$. An additional absorption associated with interband electron transitions from localized states of impurities to the Fermi level has been revealed in the $0.98-1.9-\mathrm{eV}$ spectral range. The energy bands in $\mathrm{Cu}-\mathrm{Fe}$ alloys are almost undeformed and similar to copper ones, while the Fe impurity forms localized $d$-bands at a distance of about 1.0-1.6 eV from the Fermi level. For high impurity concentrations, the experimental optical conductivity spectra $\sigma(h v)$ of the alloys are determined by a superposition of the spectra of Fe and the appropriate solid solution with the corresponding weight coefficients. The obtained experimental data allow one to calculate the density of states $N(E)$ which is related to the impurity band.
\end{abstract}

Keywords: optical conductivity, maximum solubility, Fermi level, density of states.

Manuscript received 06.08.07; accepted for publication 27.09.07; published online 31.10.07.

\section{Introduction}

The optical method is very effective for investigation of impurity states of the electron structures of alloys. This method allows one to obtain the values of parameters characterizing the impurity energy band, for example, its position in the energy spectrum and a half-width, and to calculate the density of electron states [1]. It is clear that the formation of the impurity energy bands of alloys is accompanied by essential modifications of magnetic, optical, electrical, thermal, and other properties of alloys.

According to the literature data, $\mathrm{Fe}$ is not almost dissolved in $\mathrm{Cu}$ [1]. At concentrations of $\mathrm{Fe}$ of more than $20 \%$, the heterogeneous mixture of copper with a small content of $\mathrm{Fe}$ and almost clear Fe is formed. So, the absorption spectra $\sigma(h v)$ of $\mathrm{Cu}-\mathrm{Fe}$ alloys at the indicated concentrations can be obtained by addition of the spectrum $\sigma(h v)$ of the indicated solution and that of pure Fe with fixed weights. However, it will be shown in our examinations that the absolutely other situation is observed in these alloys.

Earlier [2], the optical properties of $\mathrm{Cu}-\mathrm{Fe}$ alloys have been investigated within the narrow spectral range with the use of the constants $n$ and $\chi$ measured at ambient temperature in the photon energy range 0.18 $4.96 \mathrm{eV}$. The samples of alloys with an impurity content of $7.5,15$, and 30 at. \% were prepared by vacuum arc melting in the argon atmosphere by using several consecutive meltings. The recrystallization was performed at a pressure of $5 \cdot 10^{-6}$ Torr at $450{ }^{\circ} \mathrm{C}$ for $3 \mathrm{~h}$. We carried out the electric polishing to remove surface strains. We found that the absorption index $\chi$ and especially the refraction one $n$ increase with the concentration of $\mathrm{Fe}$, though the dependences $n(h v)$ and $\chi(h v)$ are monotone strictly increasing functions without apparent singularities. On the basis of the measured values of $n$ and $\chi$, we calculated the optical conductivity $\sigma$, whose dispersion dependences $\sigma(h v)$ for $\mathrm{Cu}-\mathrm{Fe}$ alloys and pure copper are given in Fig. 1 .

The characteristic peculiarities of the absorption spectrum $\sigma(h v)$ of all noble metals are the sharp absorption edge that corresponds to the photon energy $h v=2.1 \ldots 2.4 \mathrm{eV}$ and the very intense absorption band with a maximum at a photon energy of about 4.62$4.65 \mathrm{eV}$. It is apparent that the absorption threshold located near $2.25 \mathrm{eV}$ for pure copper is not practically displaced on the energy scale at the doping by Fe. According to the results obtained, the difference of $\sigma(h v)$ of $\mathrm{Cu}-30 \% \mathrm{Fe}$ alloy from another curve in the threshold area is related to a sufficient contribution of pure $\mathrm{Fe}$ to the absorption $\sigma(h v)$, because this alloy is an inhomogeneous mixture of the solution of $\mathrm{Cu}-15 \% \mathrm{Fe}$ 
and pure Fe. Except the absorption edge of $\sigma(h v)$ of the alloys under study in the region $2.3-3.9 \mathrm{eV}$, we observe the typical "plateau" which is formed as a result of the aliasing of some absorption bands. The intensive stable peak at $4.62-4.65 \mathrm{eV}$ in the UV region appears for all alloys, as well as for pure copper. The increase of $\sigma$ in the visible and UV regions at rising the $\mathrm{Fe}$ concentration is related, of course, to the additional absorption owing to the Fe impurity. So, in the optical spectra $\sigma(h v)$ of the investigated alloys $\mathrm{Cu}-\mathrm{Fe}$, all characteristic anomalies of $\sigma(h v)$ of pure copper become apparent and don't change their position on the energy scale at the doping by Fe. However, in the long-wave region of the spectrum (at $h v<2.0 \mathrm{eV}$ ), where interband transitions are prohibited and intraband ones are not pronounced due to high frequencies of light with $v$ as compared with the frequency of relaxation $\gamma$, the spectra $\sigma(h v)$ of alloys are significantly changed. Fig. 1 shows that, even at the $7.5 \% \mathrm{Fe}$ doping, a resonance peak of absorption with a maximum at $1.25 \mathrm{eV}$ appears in the region of $h v=$ $0.98 \ldots 1.9 \mathrm{eV}$. At a further increase in the Fe concentration, the intensity of the mentioned band strongly increase, and its peak shifts slightly (approximately by $0.10-0.12 \mathrm{eV}$ ) to the short-wave region of the spectrum. The new absorption band is not related to pure $\mathrm{Cu}$ and $\mathrm{Fe}$ and is determined by resonance states which appear in the electron spectrum of $\mathrm{Cu}$ owing to the $\mathrm{Fe}$ impurity.

The results of computer modelling have shown that it is impossible to obtain the optical spectrum $\sigma(h v)$ by a simple addition of the spectra of pure $\mathrm{Cu}$ and $\mathrm{Fe}$ or that of a solid solution $\mathrm{Cu}-2 \% \mathrm{Fe}$ and pure $\mathrm{Fe}$. On the first stage, the assumption that the optical conductivity of each alloy $\sigma_{1}(h v)$ equals the sum of the conductivities of pure $\mathrm{Cu}, \sigma_{\mathrm{Cu}}(h v)$, and $\mathrm{Fe}, \sigma_{\mathrm{Fe}}(h v): \sigma_{1}(h v)=c \cdot \sigma_{\mathrm{Fe}}(h v)+$ $(1-c) \cdot \sigma_{\mathrm{Cu}}(h v)$ (where $c$ is the concentration of $\mathrm{Fe}$ in the alloy) was made. Obviously, this relation is true in the case of the full insolubility of $\mathrm{Cu}$ and $\mathrm{Fe}$. The spectrum obtained in such a way is shown in Fig. 2.

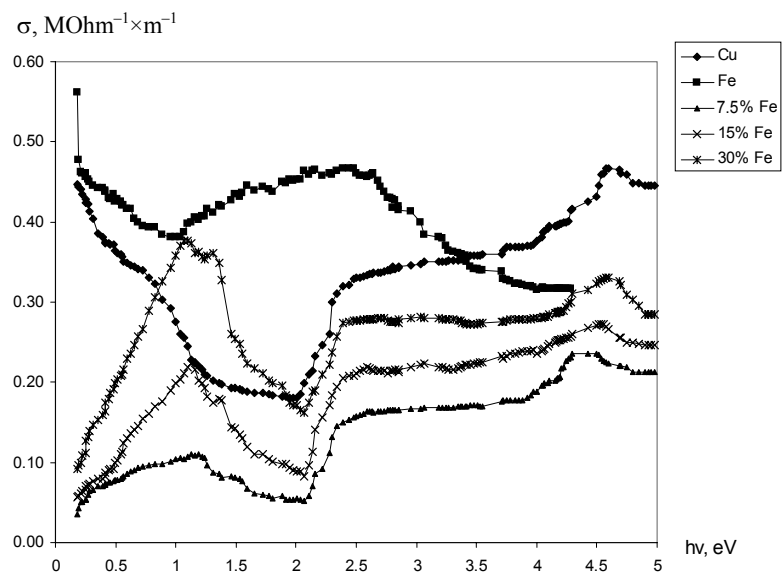

Fig. 1. $\sigma(h v)$ for $\mathrm{Cu}-\mathrm{Fe}$ alloys with $\mathrm{Fe}$ concentration $7.5,15$, and $30 \%$. (The copper curve is lifted upwards on $1.5 \mathrm{MOhm}^{-1 *} \mathrm{~m}^{-1}$.)

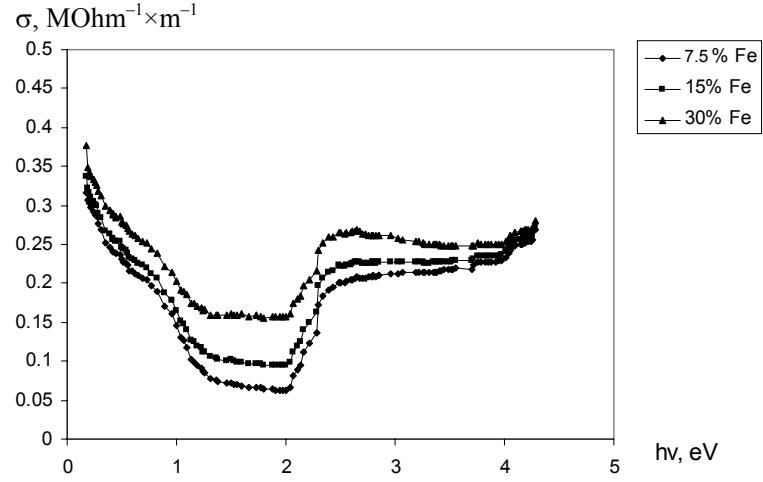

Fig. 2. Calculated curves of optical conductivity of $\mathrm{Cu}-\mathrm{Fe}$ alloys.

It is apparent that, in the visible and UV regions, the same peculiarities like in experimental curves become actual. However, in the near-IR region, the theoretical and experimental curves are strongly different. This implies that $\mathrm{Fe}$ dissolves better in $\mathrm{Cu}$, than it was considered before. Let us assume that $7.5 \% \mathrm{Fe}$ dissolves in $\mathrm{Cu}$. Then the optical spectrum of the conductivity of alloys $\sigma_{2}(h v)$ must equal the sum of conductivities of the mentioned solid solution $\mathrm{Cu}$ $7.5 \% \mathrm{Fe}, \sigma_{7.5}(h v)$, and pure $\mathrm{Fe}, \sigma_{\mathrm{Fe}}(h v)$, i.e., $\sigma_{2}(h v)=$ $c_{1} \cdot \sigma_{\mathrm{Fe}}(h v)+\left(1-c_{1}\right) \sigma_{7.5}(h v)$ (a part of the solid solution $\mathrm{Cu}-7.5 \% \mathrm{Fe}$ in the $\mathrm{Cu}-\mathrm{Fe}$ alloy). The additional absorption band at $1.6-1.9 \mathrm{eV}$ is pronounced on the theoretical curves. The intensity of this absorption band increases monotonically with the concentration of Fe, according to the experimental results, but the absorption by free carriers in the near-IR region in comparison with that of $\mathrm{Cu}$ strongly decreases. To determine how good is the correlation between theoretical and experimental curves, we consider the dispersion curves of the residual conduction $\Delta \sigma(h v)$. It was found that the value of $\Delta \sigma(h v)$ is practically independent of $h v$ and is located near zero for alloys with $7.5 \% \mathrm{Fe}$, although it strongly rises with the concentration of Fe. Our next step is the attempt to represent the optical spectrum of the conductivity of alloys $\sigma(h v)$ for a higher concentration of $\mathrm{Fe}(30 \%)$ as a sum of the conductivities of the solid solution $\mathrm{Cu}-10 \% \mathrm{Fe}$ and pure $\mathrm{Fe}$ with appropriate weights and to calculate the residual conduction $\Delta \sigma(h v)$ on their base. We found that the experimental and theoretical curves correlate well with one another for the alloy with $7.5 \% \mathrm{Fe}$ and sufficiently worse for the alloy with $30 \%$ Fe. Furthermore, the residual conduction shown in Fig. 3 is comparatively small for the alloy with $10 \% \mathrm{Fe}$ and sufficiently high for the alloys with 15 and $30 \% \mathrm{Fe}$. These results show that the dissolubility of Fe in the mentioned alloys exceeds $5 \%$.

The analysis of the data obtained indicates that it is possible to determine which changes take place in the electron spectrum of $\mathrm{Cu}$ at the doping by $\mathrm{Fe}$. The absorption edge in $\mathrm{Cu}$ is formed as a result of the electron transition from the 5-th to 6-th band, basically

(C) 2007, V. Lashkaryov Institute of Semiconductor Physics, National Academy of Sciences of Ukraine 


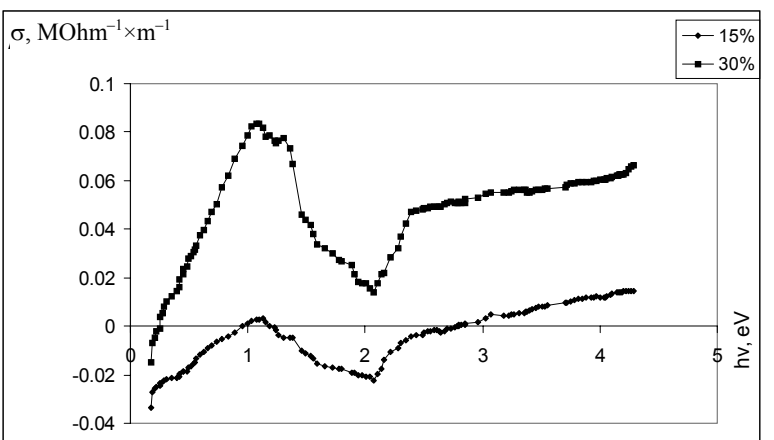

Fig. 3. $\Delta \sigma(h v)$ for $\mathrm{Cu}-\mathrm{Fe}$ alloys.

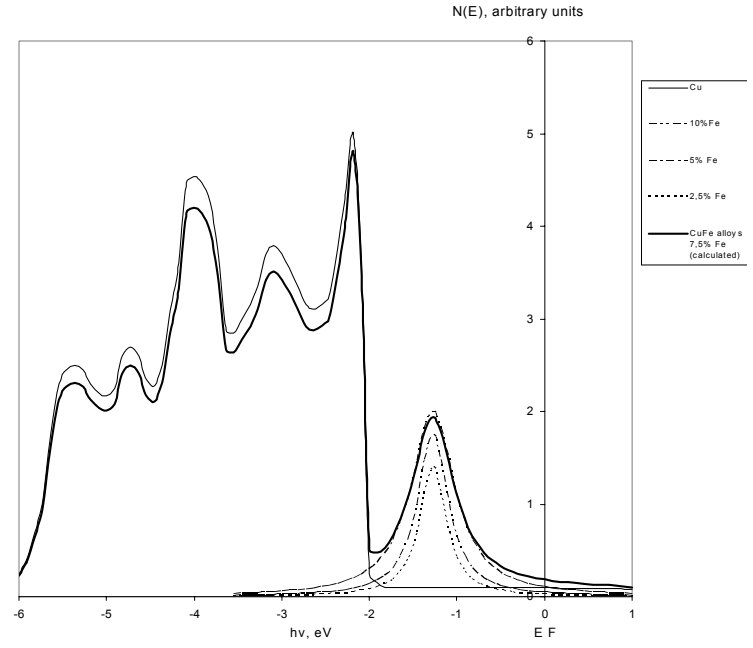

Fig. 4. Density of states for $\mathrm{Cu}-\mathrm{Fe}$ alloys.

from the peak of $d$-band $L_{3}$ on free levels of $s p$-band $L_{2}^{\prime}$ which is located near the Fermi level $E_{\mathrm{F}}$.

Experimental results show that energy gap between the peak of the $d$-band of $\mathrm{Cu}$ and the Fermi level at the doping by $\mathrm{Fe}$ remains invariable, i.e., the location of $d$ bands compared to the Fermi level $E_{\mathrm{F}}$ in the electron spectrum of alloys doesn't change. Above the absorption edge, the intensive interband absorption is observed. It corresponds to transitions in the large volume of the Brillouin zone from the 2-nd, 3-rd, and 4-th bands on free states of the 6-th band in $\mathrm{L}-\mathrm{W}$ and $\Gamma-\mathrm{X}$ directions. As the character of the spectrum $\sigma(h v)$ doesn't change at the doping by $\mathrm{Fe}$ in the energy region $h v=$ $2.5 \ldots 4.0 \mathrm{eV}$, this means that $d$-bands which are located below the Fermi Level $E_{\mathrm{F}}$ by $2.5-4.0 \mathrm{eV}$ are not deformed strongly in alloys. Since the high-energy peak on the curve $\sigma(h v)$ for $\mathrm{Cu}$ at $4.7 \mathrm{eV}$ is related to transitions from the 6-th to 7-th band, basically from $d$ states $L_{1}$ on free $s p$-levels of $L_{2}^{\prime}$ band, we obtain that the structure of the $d$-bands located by $4.0-4.95 \mathrm{eV}$ below the Fermi level is not changed and similar to the $d$-bands of $\mathrm{Cu}$. Energy bands in $\mathrm{Cu}-\mathrm{Fe}$ alloys keep a characteristic pattern of the electron spectrum of pure copper. However, the appearance of a new absorption band at $1.10-1.15 \mathrm{eV}$ in the optical spectrum $\sigma(h v)$ of $\mathrm{Cu}-\mathrm{Fe}$ alloys testifies that an additional $d$-subband, which is related to the Fe impurity, is formed in the electron spectrum. Let us assume that the new absorption band is formed as a result of the electron transitions from the impurity $d$-band to free stated located in the region of the Fermi level $E_{\mathrm{F}}$. Then it is possible to calculate the density of states $N(E)$ on the basis of the obtained experimental data which are related to the impurity band $[4,5]$. To calculate $N(E)$, it is necessary to determine the energy distance of the impurity band center from the Fermi level, $E_{d}-E_{\mathrm{F}}$, and its half-width $\Delta$ which are 1.25 and $0.3 \mathrm{eV}$ for $\mathrm{Cu}$ $7.5 \% \mathrm{Fe}$ as well as 1.13 and $0.41 \mathrm{eV}$ for $\mathrm{Cu}-15 \% \mathrm{Fe}$ accordingly to experimental data.

The density of states $N(E)$ for $\mathrm{Cu}$ and the contribution to the density of states of $\mathrm{Cu}-\mathrm{Fe}$ alloys with $2.5,5$, and $10 \% \mathrm{Fe}$ from the impurity band are shown in Fig. 4. It is possible to approximately consider that the complete density of states $N(E)$, for example, for $\mathrm{Cu}$ $5 \% \mathrm{Fe}$ alloy, is a sum of the densities of states for both pure copper and the impurity $d$-band of Fe.

The above-presented results testify that a single band of conductivity of alloys that keeps characteristic patterns of the electron spectrum of $\mathrm{Cu}$ is formed as a result of the interaction of the $s p$ - and $d$-bands of $\mathrm{Cu}$ and $d$-bands of Fe. An additional $d$-band which is located between the Fermi level and the peak of $d$-bands of $\mathrm{Cu}$ is formed owing to the $\mathrm{Fe}$ impurity, which does not contradict the data on the temperature behavior of thermal, electrical, and magnetic properties of the mentioned alloys.

\section{References}

1. V.S. Staschuk, S.N. Tkachenko, A.E. Doroshenko // Fizika Metalov Metallovedenie 62, p. 830 (1986) (in Russian).

2. H. Ehrenreich, L.M. Schwartz, The Electronic Structure of Alloys. Academic Press, New York, 1976.

3. L.V. Poperenko, V.S. Staschuk, V.V. Vovchenko // Funct. Mater. 7, p.482-487 (2000).

4. V.S. Staschuk, S.N. Tkachenko, M.N. Yasinskaya // Zhurnal Prikladnoy Spectroskopii 44, p. 634-639 (1986) (in Russian).

5. V.S. Staschuk // Ukrainsky Fizychny Zhurnal 41, 675 (1996) (in Ukrainian). 